Preprint: Final version appears as:

Morgan, R., Freudiger-Bonzon, J., Nichols, K., Jellis, T., Dunkerley, S., Zelazowski, P. \& Bull, P.

(2009) The forensic analysis of sediments recovered from footwear. In Ritz, K, Dawson, L \& Miller,

D. (Eds) Criminal and Environmental Soil Forensics. e-ISBN 978-1-4020-9204-6

\title{
The forensic analysis of sediments recovered from footwear
}

Morgan, R. M., Freudiger-Bonzon, J., Nichols, K. H., Jellis, T., Dunkerley, S., Zelazowski, P. and Bull, P. A.

Communicating author: Ruth Morgan

Ruth.morgan@ucl.ac.uk

Tel: +442031083037

Fax: +442031083088 
The forensic analysis of sediments recovered from footwear

Morgan, R. M., Freudiger-Bonzon, J., Nichols, K. H., Jellis, T., Dunkerley, S.,

Zelazowski, P. and Bull, P. A.

\begin{abstract}
The forensic analysis of sediments recovered from footwear has the potential to yield much useful information concerning the movements of a person before, during and after a crime has taken place. Three experimental studies and a number of examples of forensic casework provide insight into the complexity of the spatial distribution of geoforensic materials on the soles of footwear and the persistence of these materials over time on the soles and uppers. These findings have implications for both the geoforensic sampling protocols and procedures for footwear submitted for analysis in a criminal investigation and also for the analysis of any materials recovered.
\end{abstract}

The preservation of sediment on a shoe sole will vary, with certain areas generally retaining more sediment than others. The sequential layering of sediments that have been transferred to the shoe will be preserved in some cases and in certain areas, but generally undergoes complex mixing. Such mixing of sediment from different sources occurs both across the shoe sole and also through time, it is therefore, important to be aware of these variations when taking samples for analysis if representative samples are to be taken and meaningful interpretation of any analysis derived is to be effected. Furthermore, such mixing of pre-, syn- and post-forensic event sources has implications for the appropriateness of different analytical techniques. Visual identification techniques which are able to identify where such mixing has taken place are preferred to forms of analysis that require homogenisation of the sample prior to analysis as this reduces the possibility of false-negative or positive associations when undertaking comparison of samples in a forensic context. The context within which any sampling or analysis is undertaken is crucial for a meaningful and accurate interpretation of the geoforensic evidence 


\section{INTRODUCTION}

The forensic analysis of soils and sediments is a rapidly developing field that has its roots in the geosciences and which applies geoscience principles to the forensic arena (Morgan and Bull 2007a and b). The underlying premise in geoforensic study is that evidence will be transferred from sources to recipient mediums (such as clothing, vehicles etc.) The concept that 'every contact leaves a trace' was first articulated by Locard $(1928,1930)$ and these ideas have been developed more recently by Inman and Rudin (2002). The analysis and interpretation of geoforensic evidence also draws upon the body of literature concerning other forms of trace physical evidence, particularly with regard to the nature of evidence transfer and persistence (for example, Wiggins et al. 2002, Roux et al. 1999, Hicks et al. 1996, Pounds and Smalldon 1975a, b, c).

The deposits found on and in footwear have proved an attractive source of comparator samples in geoforensic studies. Since footwear is in contact with the ground, there appears to be a reasonable opportunity to compare materials from the footwear with pertinent scenes related to a forensic event. The reality is however, far more complicated - the devil is in the detail: Firstly, the very transfer of materials onto the soles of footwear will vary in relation to a number of physical characteristics (grain type and size, organic content; Chazottes et al. 2004, Virtanen et al. 2007) and secondly, the transfer of materials is rarely onto a surface that does not already hold materials which may have been deposited before the forensic event in question. Likewise, materials on, or in, a shoe may derive from sources encountered after the particular forensic event and thus, there may be at least three phases of sediment transfer which may themselves not be evenly distributed across the sole or upper of the shoe. Once transferred to footwear, the absolute amount of trace material will start to decrease and the persistence of such materials becomes a very relevant consideration. Paradoxically, the longer material is able to survive on footwear, the more problematic the interpretation of such evidence may become. However, if the material does not persist long enough to be collected it will not be of any evidential value and will in turn impact upon the results of the crime reconstruction. 
Sampling procedures, whether for physical, chemical or biological analyses must take into account these spatial and temporal considerations since the resultant interpretation will depend heavily upon these previous constraints (Morgan and Bull 2007b). Whether it is possible to identify an exclusion or to differentiate a falsepositive exclusion is a matter that is considered herein. Further, we address a number of other pertinent issues relating to the collection, analyses and interpretation of geoforensic evidence on footwear which fall broadly within the themes of spatial distribution and persistence.

The spatial considerations are; whether sequentially deposited layers of material are preserved in the chronological order in which they were transferred to the footwear sole; whether mixing (from pre-, syn- and post-forensic event) takes place on the sole of footwear in an ordered or more random manner; and whether sampling vertically or horizontally through the sediment deposit on the sole is affected by sample mixing and layer distortion.

The temporal (persistence) considerations are whether geoforensic evidence transferred onto footwear (both the upper and sole) survives for a sufficient period of time for subsequent collection and analyses.

\section{SPATIAL MIXING OF SOIL AND SEDIMENT ON FOOTWEAR}

\subsection{Experimental studies}

Contact with the ground almost inevitably results in the transfer of materials onto the sole (or uppers) of footwear (one-way transfer), but may also initiate the transfer of material on the footwear to the underlying surface thus initiating two-way transfer (Locard 1930). Such transfers have been reported and analysed in the published literature in both experimental and case work studies (Bull et al. 2006, Morgan et al. 2006, Morgan and Bull 2006, Bull et al. 2004, Horrocks et al. 1999).

Recent experimental work has concentrated on the problem of discrete sampling of mud on footwear on the soles of footwear by studying the distribution and movement of three layers of (Plasticine ${ }^{\mathrm{TM}}$ ) during experimental runs which involved the wearer of the shoes walking and running. Plasticine was chosen as a soil proxy particularly 
of clay materials which constitute an important component of many soils. Although lacking the coarse silt and sand component of many soils, the Plasticine appeared to mimic results observed in case work described here in section 2.2. Forthcoming papers deal with the mixing of three different soil types the result of which are beyond the remit of this paper. Here, three different colours of Plasticine were chosen to represent pre-, syn- and post-forensic event sources of soil. All three layers of Plasticine (the same size and thickness $(5 \mathrm{~mm})$ ) were applied sequentially to the soles of identical pairs of flat soled training shoes. Following the application of each layer, the wearer walked $250 \mathrm{~m}$ on paved ground in dry conditions so that layer 1 was eventually walked on for $750 \mathrm{~m}$, and layers 2 and 3 were walked on for $500 \mathrm{~m}$ and $250 \mathrm{~m}$ respectively. The experiment was replicated under the same conditions. A further experiment was undertaken which followed the same procedure, the only difference being that the wearer ran on each layer of Plasticine. A template of each shoe was constructed (using a model derived by Hessert et al. 2005) and sampling points (on a grid system) were identified. Vertical plugs of the Plasticine remaining on the sole of the training shoes were taken at each sample point for subsequent analysis. These plugs were then photographed in cross-section and the resultant digital images were pixelated using MATLAB to provide numerical comparison of the proportion of each layer (by colour) of Plasticine preserved at each sampling point. The general results for the right shoe from each experiment (both walking and running) are presented in figure 1 and more detailed presentation can be seen in figure 2.

Visual inspection of both figures shows that the three layers on the right shoe (layer 1 was yellow, layer 2 was blue and layer 3 was red) although originally of the same thickness, now comprise layers of different thicknesses for both the experiments involving walking and running (figure 1) with red the predominant colour preserved in each plug. Spatial variation of the Plasticine on the right shoe (figure 1) shows a predominance of the last layer applied (red) and this is shown in figure 2 where the relative area proportions of each layer are presented for the medial arch area of the shoe (MA) and for the toes area of the shoe (T) (for location of MA and T see figure $1)$. 
The spot sampling of footwear in order to compare a footwear sample with a sample from a forensic site requires an assumption that the area of footwear sampled provides an accurate comparator for materials derived from a forensic site. If our forensic event is the middle layer of the three presented in figures 1 and 2, then the blue layer (layer 2) assumes great importance. Multivariate statistical analysis (by canonical discriminant analysis) of the 'toe' (H, T, MT1, 3 and 4) and 'middle' area (MA and LA) of the shoe show that these two areas can not be discriminated (Wilks lambda $=$ 0.996, $\mathrm{p}=0.89$ thus $\mathrm{p}>0.05$ ). However, when the 'heel' area (MC and LC) was included in the statistical analysis, the three designated areas of the shoe sole (toe, middle and heel) could be discriminated from each other at the $99 \%$ significance level (Wilks lambda $=0.761, \mathrm{p}=0.001$ thus $\mathrm{p}<0.01$ ). There is therefore a statistically significant difference between the layers preserved in the different spatial areas of the footwear sole. These results have dramatic implications for the production of untestable false-positive or false-negative associations between materials taken from the sole of a shoe and materials taken from a site of forensic interest, especially when general sole samples are taken or analysis of the materials requires homogenisation (such as when using chemical analysis (such as Inductively Coupled Plasma Spectrometry (ICP)) or certain physical analyses (colour, particle size etc.)). Such caution has been suggested in the geoforensic literature in relation to geoforensic analysis more generally (Morgan and Bull 2007a, Morgan and Bull 2007b). It becomes critical therefore, that the establishment of sample procedures and protocols occurs and that they are adopted when investigations of sediments on the soles of footwear are used in forensic investigation (see below in section 2.2)

Specific rheological observations of the right shoe under walking or running conditions (figure 2) show differences between the movements of the three layers depending upon the movement of the wearer. During the walking experiment, the sequential chronology of the three layers appears to be broadly preserved in both the MA and $\mathrm{T}$ sections of the sole. In contrast, after running, mixing appears to occur amongst the layers, disrupting their chronological sequence, indeed, in the medial arch area (MA) of the sole of the footwear, layers 1 and 2 (yellow and blue) are broadly removed from the area leaving layer 3 (red) as the sole or predominant material present. This will also have severe implications for the sampling and subsequent analysis of sediment recovered from footwear. If mixing is occurring it is 
suggested that analytical techniques are used which can identify the different layers even if they have undergone significant mixing with sediment from different sources.

The experiment above highlights the problems of spot sampling of a shoe, the necessity to avoid homogenisation of samples prior to analysis, and the importance of visual rather than automated forms of analyses (particularly when this analysis requires homogenisation of the sample). Interpretations of the results are further complicated by the possible false positive or negative association between the sample from the footwear or the sole of the footwear with that of the desired forensic event area. Some practitioners recognise some of these problems and choose to take various samples from the soles of footwear submitted for analysis. There appears to be no fixed protocol and only suggestion in the literature with for example, Murray and Tedrow (1975) who advocate the methods of Georg Popp in the need for sequential layer analysis to reconstruct the pre-, syn- and post-forensic event history of mud deposition on the sole of footwear.

\subsection{Casework example}

This example provides a case study of the prosecution of a man accused of digging up a badger sett in the Oswestry area of central England. Fundamental to the case was the comparison of soils taken from a pair of boots, two shovels and the much disturbed dug-out badger sett site. The soil exhibit taken from the badger sett site comprised hundreds of grams of sediment whilst the sample recovered from the shovels comprised only tens of grams and the footwear only a few grams of material. In order to overcome the problem of comparison between exhibits, a number of spot samples were taken from each exhibit (see figure 3 and for further details Morgan et al. 2006).

The physical (colour, particle size analysis, quartz grain surface texture analysis) and chemical analyses (conductivity, pH, Atomic Absorption Spectromety and Dionex (AAS/Dionex)) undertaken on these soil samples is documented in Morgan et al. (2006); none of these techniques were able to discriminate between the soil samples taken from the boots with the soil samples taken from the badger sett site. Further analysis of the quartz grain surface textures revealed that the quartz component of the 
soil was made up of three distinct types of grain. Type I grains were characterised as deriving from a diagenetic sandstone exhibiting a suite of diagenetic features including both anhedral and euhedral crystal growth typically without subsequent edge abrasion (figure 4A and B). Type II grains were well rounded grains with subaqueous impact features (such as found after river transportation) with later chemical smoothing (Figure 4C and D). Type III grains were characteristically high relief with angular/subangular grains with some subrounded additions with no edge abrasion but later chemical smoothing (Figure 4E and F. The grains were classified according to the system designated by Bull and Morgan (2006) from which it can be seen that only $0.5 \%$ of the quartz grains included in the database derived from English forensic soil samples (approximately 35,000 grains) were of the same form as Type I identified here. The palaeoenvironmental assessment of these soils is that the Type I and III grains derive from the local sandstone, having not been transported by wind or water and thus exhibiting no grain edge abrasion, whilst Type II grains represent most likely a fluvial input into the area and mixing on site with the Type I and III grains. This very limited assemblage of quartz grain types makes the similarity of the materials found in samples taken from both boots and the badger sett site significant. Indeed, multivariate statistical analysis (canonical discriminant function analysis) demonstrated that it was not possible to discriminate between the quartz grain type assemblages from each location presented in Table 1 (Wilks lambda $=0.685$, $\mathrm{p}=0.225$ ). In this case, it was not possible to exclude the soil samples taken from the footwear from the soil samples taken from the badger sett site.

In order to come to a meaningful interpretation, it was necessary to take multiple samples (in this case 22 spot samples were able to be collected) from the sole of the footwear to compare with multiple samples (in this case10 samples) taken from the bulk soil sample recovered from the badger sett site. It was not merely an analysis involving two comparator samples; over 30 samples were eventually analysed. It must also be stressed that this analysis represented only one strand of forensic investigation which itself utilised geoforensic results taken from independent techniques such as pollen analysis and this was undertaken by separate scientists who worked independently in this case.

Various aspects of the complexity of the spatial distribution and movement of pre-, syn- and post-forensic event soil on the soles of footwear have been identified in these 
experimental and casework studies. Footwear often provides the starting point for geoforensic enquiry, but this work highlights the need for caution when making comparison between soil samples recovered from the soles of footwear and forensic sites. There is no such thing as simple comparison nor is there any philosophical basis for attempting to 'match' samples (Morgan and Bull 2007b, Bull et al. in press).

\section{PERSISTENCE OF TRACE EVIDENCE ON FOOTWEAR}

\subsection{Experimental Studies}

The long-standing view that trace materials persist on clothing stems from the experimental works undertaken by Robertson and Roux (2000), Hicks et al. (1996) Pounds and Smalldon (1975a, b, c) where studies have generally provided decay curves of 4-8 hours duration. More recent work has sought to extend the experimental decay curve timeline to hundreds of hours in an attempt to utilise the power of electron microscopy (Bull et al. 2006). The experiments presented herein deal with the quantities of materials left on the soles of footwear over time, and the persistence of pollen particulates on the uppers of shoes over even longer periods of time.

The obvious advantages of finding trace particulates on clothing and footwear many hours after their transfer at the relevant forensic event could be argued to be outweighed by the very problems of persistence where the picture is complicated by pre-, syn- and post-event mixing. These problems, similar to those experienced in section 2, are best overcome with resort to visual identification methodologies.

\subsubsection{The persistence of trace materials on the soles of footwear through time} An experimental study was undertaken to establish the nature of the persistence of silt-sized trace materials on the soles of footwear over time. In order to quantify the amount of trace sediment present, a UV powder $(<15 \mu \mathrm{m})$ was mixed with soil and applied to the soles of training shoes. At a number of intervals (after 0m, 100m, $250 \mathrm{~m}, 350 \mathrm{~m}, 450 \mathrm{~m}$ ) the sole of each training shoe was photographed under an ultraviolet light. This digital image was then pixelated in IDRISI to provide an indication of the amount of silt-sized material remaining on the sole. This experiment was 
repeated three times and the results (figures 5 and 6) show a general trend of an initial reduction in the amount of trace material adhering to the sole after $100 \mathrm{~m}$ of walking on a smooth concrete surface. However, after $250 \mathrm{~m}$ the amount of silt-sized material has increased with a subsequent decrease after $350 \mathrm{~m}$. This pattern is due to the larger conglomerated materials decaying rapidly during the initial stages of walking. However, the remaining material is then spread out by the pressure applied through the foot during subsequent walking which in turn increases the distribution of siltsized material. After this stage the trace material again rapidly decays, however, it is interesting to note that a sufficient amount of material remains on some parts of the soles for sampling to take place even after $450 \mathrm{~m}$ of walking.

\subsubsection{The persistence of pollen particulates on the uppers of footwear over time}

In a second study, two pairs of shoes (a cotton plimsoll and a suede shoe) were used to assess the transfer and persistence of pollen particulates on the uppers of footwear. The participant brushed past a flowering shrub (Jasminium Nudiflorum) wearing each pair of shoes and then wore the shoes for a total of seven days undertaking general activities. Tapings were taken from pre-marked locations (to prevent repeat sampling) across the toe of each shoe at different time intervals $(0,2,4,6,8,10,12$, 24, 36 and 168 hours) and observed under a scanning electron microscope at x330 where the pollen grains present were counted. The results of each replicated experiment are presented in figures $7 \mathrm{a}$ and $\mathrm{b}$.

The persistence of pollen on these two different types of footwear appears to follow the previously identified trend of two/three stage decay (Pounds and Smalldon 1975a, b, c, Bull et al. 2006). The loss of pollen is particularly rapid during the first four hours, with subsequently less rapid loss between 4 and 10 hours, followed by a period of much slower decay. After 168 hours, $4.8 \%$ and $1.5 \%$ of the original pollen remained on the suede shoes whilst 1.8 and $1.0 \%$ remained on the cotton plimsolls. Whilst these appear to be only small percentages of remaining pollen, they are only taken from a very small area of the shoe upper (approximately $0.5 \mathrm{~cm}^{2}$ ). These results therefore, have important implications for the forensic examinations of footwear; pollen particulates are highly likely to persist for many hours, days or even weeks. Such evidence has great potential to aid criminal investigations (Mildenhall 2006, Mildenhall et al. 2006, Horrocks et al. 1999, Horrocks and Walsh 1999) and this 
present study demonstrates that pollen is likely to remain on footwear for significant periods of time during normal wear thus enabling its recovery and analysis. Indeed this phenomenon makes the likelihood much greater of pre and post-forensic event mixing with the relevant materials taken from the forensic scene. Thus the quality of persistence has both 'advantages' and 'disadvantages' for the interpretation of forensic evidence.

\subsection{Case Studies}

The experimental studies outlined above have demonstrated that geoforensic evidence has the potential to persist on footwear (both soles and uppers) for significant periods of time. As mentioned previously, this not only has implications for the recovery and sampling of such evidence from footwear, but also has implications for the type of analytical technique employed to analyse such evidence. If trace geoforensic evidence persists for long periods of time, it will be important (as mentioned in section 2) that materials from different sources can be identified during any subsequent analyses.

In our experience there have been a number of criminal investigations that have utilised the presence and recovery of geoforensic evidence from footwear. In the case of R v Wren (2002) distinctive gravel was recovered from the footwear sole of a suspect which could not be excluded from having derived from the crime scene. Similarly, in R v Hunt and Fawley (2002) a number of pairs of shoes were seized by the police and the comparison of trace geoforensic evidence recovered from the footwear and the crime scene enabled all but two pairs of footwear to be excluded from the investigation which aided the police in their crime reconstruction.

It is, however, not always evidence collected directly from footwear that can aid criminal enquiry. In the case of $\mathrm{R} v$ Flavious (2005) a car was used to transport a body to a grave site and then driven back to the home of the suspect. The car was seized and the driver footwell mat was found to be very muddy, with one distinct footprint present (see figure 8). The general debris was sampled and the mud from the footprint also collected and compared to the soil sampled from the body deposition site. Additionally, a pair of boots were seized which had mud present on the soles. A number of analytical techniques were employed (elemental chemistry, 
mineralogy, colour, $\mathrm{pH}$, particle size analysis) in addition to the quartz grain surface texture analysis. A distinctive quartz grain 'type' was identified in the samples collected from the car footwell and the body deposition site but it was conspicuous in its absence in the boot samples. Another search at the home of the suspect yielded a pair of training shoes which had been washed in a washing machine. Whilst this footwear appeared to be very clean, inspection of the inners of the shoes underneath the in-sole yielded a small amount of clean debris. The quartz grains were analysed and the distinctive grain type was found to be present. In this instance, it was the geoforensic evidence deposited by the footwear that proved to be very important as well as the evidence contained within it. Furthermore, due to the mixing of sediment from different sources in the car footwell and in the training shoes it was necessary to employ a visual technique (in this case, quartz grain surface texture analysis see Bull and Morgan 2006 and section 2.2) that was able to identify this mixing to be more confident of the interpretation of the analysis and avoid false-exclusionary conclusions.

Thus footwear has the potential to provide very useful information during the course of a forensic investigation. Not only the sediment transferred and preserved on the footwear (both inners and outers, uppers and sole) but also the sediment deposited as muddy footprints or collected by a plaster cast of footprint impression (see Bull et al. 2006) can be recovered and provide valuable contextual information.

\section{CONCLUSIONS}

The implications of these experimental studies and case work examples are twofold and have a bearing upon both sampling and analysis of geoforensic evidence recovered from footwear. In terms of sampling footwear for geoforensic evidence we have shown that certain areas of the sole are more likely to retain sediment than others. Of the soil/sediment evidence that is retained, it is unlikely that a sequential chronology of the different sources of sediment will be preserved; mixing of evidence from different sources does occur (in some areas more than others) on the soles of footwear.

Geoforensic evidence has been shown to persist for reasonable periods of time on the soles of footwear (section 3.1.1) and the uppers (section 3.1.2) as well as after 
washing in the inners of shoes (section 3.2). The persistence of geoforensic evidence on footwear means that it is likely to be present and should be sampled to see if any such evidence can be recovered. However, it also means that the analytical technique employed must be able to identify when mixing of evidence from different sources which has been introduced at different stages has occurred if meaningful interpretation of the analysis is to be made. Indeed, the spatial variation of geoforensic evidence preserved on the sole of a shoe also means that it is crucial to employ visual techniques that are able to identify materials derived from different sources. It is vital however, that a proper preliminary optical examination initially under low magnification be conducted before more advanced analytical procedures are employed. Presently the developments of digital photography which enable high magnification and resolution provide excellent opportunity for preliminary visual analysis. Identification of materials derived from different provenances is of great importance because if different sources of geoforensic material are not identified there is a real possibility for un-testable false negative interpretations of the evidence to be reached. Such un-testable conclusions can have no place in a court of law.

Indeed, this paper also serves to highlight the importance of experimental studies for deepening our knowledge and increasing our ability to interpret forensic evidence accurately and meaningfully within the appropriate context. Such studies have a valuable role to play in the development of forensic science (Morgan et al. in press).

\section{REFERENCES}

Bull, P. A., Morgan, R. M., Wilson, H. E. and Dunkerley, S. (2004) Multi-technique comparison of source and primary transfer soil samples: an experimental investigation'. A comment. Science and Justice 44(3):173-176.

Bull, P. A. and Morgan, R. M. (2006) Sediment Fingerprints: A forensic technique using quartz sand grains. Science and Justice 46(2):64-81

Bull, P. A., Morgan, R. M., Sagovsky, A. and Hughes, G. J. A. (2006) The Transfer and Persistence of Trace Particulates: Experimental studies using clothing fabrics. Science and Justice. 46(3):182-191

Bull, P. A., Morgan, R. M. and Freudiger-Bonzon, J. (in press) A critique of the present use of some geochemical techniques in geoforensic analysis. Forensic Science International. (available online) 
Bull, P. A., Parker, A. J. and Morgan, R. M. (2006) The forensic analysis of soils and sediment taken from the cast of a footprint. Forensic Science International 162:6-12

Chazottes,V., Brocard, C. and Peyrot, B. (2004) Particle size analysis of soils under simulated scene of crime conditions: the interest of multivariate analyses. Forensic Science International 140, Issues 2-3 :159-166.

Hessert, M. J., Vyas, M., Leach, J., Hu,K., Lipsitz, L. A. and Novak, V. (2005) Foot pressure distribution during walking in young and old adults, BMC Geriatrics 5: 8 Hicks, T., Vanina, R. and Margot, P. (1996) Transfer and Persistence of Glass Fragments on Garments. Science and Justice 36 (2): 101-107.

Hicks, T., Vanina, R. and Margot, P. (1996) Transfer and Persistence of Glass Fragments on Garments. Science and Justice, 36 (2): 101-107

Horrocks, M., Coulson, S. A. and Walsh, K. A. J. (1999) Forensic palynology: variation in the pollen content of soil on shoes and in shoeprints in soil. Journal of Forensic Science 44 (1) 119-122.

Horrocks, M. and Walsh (1999) Fine resolution of pollen patterns in limited space: Differentiating a crime scene from an alibi scene seven metres apart. Journal of Forensic Science 44(2) 417-420.

Inman, K. and Rudin, N. (2002) The origin of evidence. Forensic Science International $126 \mathrm{p} 11-16$

Locard, E. (1928) Dust and its analysis, Police Journal 1, 177

Locard, E. (1930) Analyses of dust traces parts I, II and III. American Journal of Police Science 1: 276-298, 401-418 and 496-514.

Mildenhall, D. C. (2006) Hypericum pollen determines the presence of burglars at the scene of a crime: An example of forensic palynology. Forensic Science International 163(3):231-235

Mildenhall, D. C., Wiltshire, P. E. J. and Bryant, V. M. (2006) Forensic Palynology: Why do it and how it works. Forensic Science International 163(3): 163-172

Morgan, R. M., Allen, E, Lightowler, Z., Freudiger-Bonzon, J. and Bull, P. A. (in press) A Forensic Geoscience Framework and Practice. Policing: A Journal of Policy and Practice.

Morgan, R. M. and Bull, P. A. (2006) Data interpretation in forensic sediment geochemistry. Environmental Forensics 7(4):325-334

Morgan, R. M., Wiltshire, P., Parker, A. and Bull, P.A. (2006) The Role of Forensic Geoscience in Wildlife Crime Detection. Forensic Science International 162: 152162 
Morgan, R. M. and Bull, P. A. (2007a) The philosophy, nature and practice of forensic sediment analysis. Progress in Physical Geography. 31(1):43-58

Morgan, R. M. and Bull, P. A. (2007b) Forensic Geoscience and Crime detection. Identification, interpretation and presentation in forensic geoscience. Minerva Medicolegale 127 (2): 73-90

Murray, R. C. and Tedrow, J. C. F. (1975) Forensic Geology: Earth Science and Criminal Investigation. Rutgers University Press.

Pounds, C. A. and Smalldon, K. W. (1975a) The transfer of fibres between clothing materials during simulated contacts and their persistence during wear, part I - Fibre transference. Journal of the Forensic Science Society 15: 17-27.

Pounds, C. A. and Smalldon, K. W. (1975b) The transfer of fibres between clothing materials during simulated contacts and their persistence during wear, part II. Journal of the Forensic Science Society 15: 29-37

Pounds, C. A. and Smalldon, K. W. (1975c) The transfer of fibres between clothing materials during simulated contacts and their persistence during wear, part III - a preliminary investigation of the mechanisms involved. Journal of the Forensic Science Society 15: 197-207

Robertson, J. and Roux, C. (2000) Transfer and Persistence. In Siegel, J. A., Saukko, P. J. and Knupfer, G. C. (eds) Encyclopedia of Forensic Sciences. Academic Press, pp. $834-838$

Roux C., Langdon S., Waight D., Robertson J., (1999) The Transfer and Persistence of Automotive Carpet Fibres on Shoe Soles. Science \& Justice 39(4), 239-251;

Virtanen, V., Korpelainen, H., Kostamo, K. (2007) Forensic botany: Usability of bryophyte material in forensic studies Forensic Science International 172 (2-3): 161163

Wiggins, K. G., Emes, A. and Brackley, L. H. (2002) The transfer and persistence of small fragments of polyurethane foam onto clothing. Science and Justice 42:105-110 


\section{List of Figures and Tables}

Figure 1 Plasticine plugs recovered from each sample point on the right shoe sole for both walking and running experiments $(\mathrm{MC}=$ medial calcaneus, $\mathrm{LC}=$ lateral calcaneus, $\mathrm{MA}=$ medial $\operatorname{arch}, \mathrm{LA}=$ lateral $\operatorname{arch}, \mathrm{MT1}=$ first metatarse, 3 = second and third metatarse, 4 = fourth and fifth metatarse, $\mathrm{H}=$ hallux, and $\mathrm{T}=$ toes).

Figure 2 The percentage of each layer comprising the plasticine plugs recovered from the medial arch area and the toes area of the right shoe soles for both walking and running experiments.

Figure 3 The sampling points on the soles of the boots submitted for analysis.

Figure 4 Quartz grain types found in samples taken from the badger sett site and boots submitted for analysis; A and B Type I grains, C and D Type II grains, E and F Type III grains.

Figure 5 Pixelated image to show the silt-sized material retained on the shoe soles after walking different distances. (Mean brightness indicates the amount of silt-sized material remaining on the sole).

Figure 6 Figure 6 Graph to show the general trend in mean pixel brightness (proxy for amount of silt-sized sediment) on the soles of footwear over distance $(n=6)$

Figure 7a Graph to show the persistence of pollen particulates on the suede shoes over time.

Figure $7 \mathrm{~b}$ Graph to show the persistence of pollen particulates on the cotton shoes over time.

Figure 8 Photograph to show the muddy footwell mat and the distinct footprint

Table 1 Quartz grain types identified in the soil samples taken from the crime scene and the boots 


\begin{tabular}{|c|c|c|c|c|c|}
\hline \multicolumn{2}{|c|}{ Case Samples } & $\begin{array}{l}\text { Type } \\
\text { I }\end{array}$ & $\begin{array}{l}\text { Type } \\
\text { II } \\
\end{array}$ & $\begin{array}{l}\text { Type } \\
\text { III }\end{array}$ & Total \\
\hline \multirow{10}{*}{$\begin{array}{l}\text { Badger } \\
\text { sett site }\end{array}$} & Sub-sample 1 & 41 & 19 & 7 & 67 \\
\hline & Sub-sample 2 & 43 & 15 & 3 & 61 \\
\hline & Sub-sample 3 & 47 & 7 & 3 & 57 \\
\hline & Sub-sample 4 & 41 & 9 & 3 & 53 \\
\hline & Sub-sample 5 & 48 & 14 & 1 & 63 \\
\hline & Sub-sample 6 & 38 & 12 & 2 & 52 \\
\hline & Sub-sample 7 & 66 & 11 & 4 & 81 \\
\hline & Sub-sample 8 & 47 & 13 & 6 & 66 \\
\hline & Sub-sample 9 & 28 & 10 & 5 & 43 \\
\hline & Sub-sample 10 & 35 & 8 & 4 & 47 \\
\hline Right Boot & composite & 34 & 16 & 6 & 56 \\
\hline \multirow{4}{*}{ Left Boot } & composite & 29 & 14 & 1 & 44 \\
\hline & point 1 & 38 & 5 & 6 & 49 \\
\hline & point 2 & 34 & 5 & 8 & 47 \\
\hline & point 3 & 37 & 13 & 6 & 56 \\
\hline
\end{tabular}

Table 1 Quartz grain types identified in the soil samples taken from the crime scene and the boots

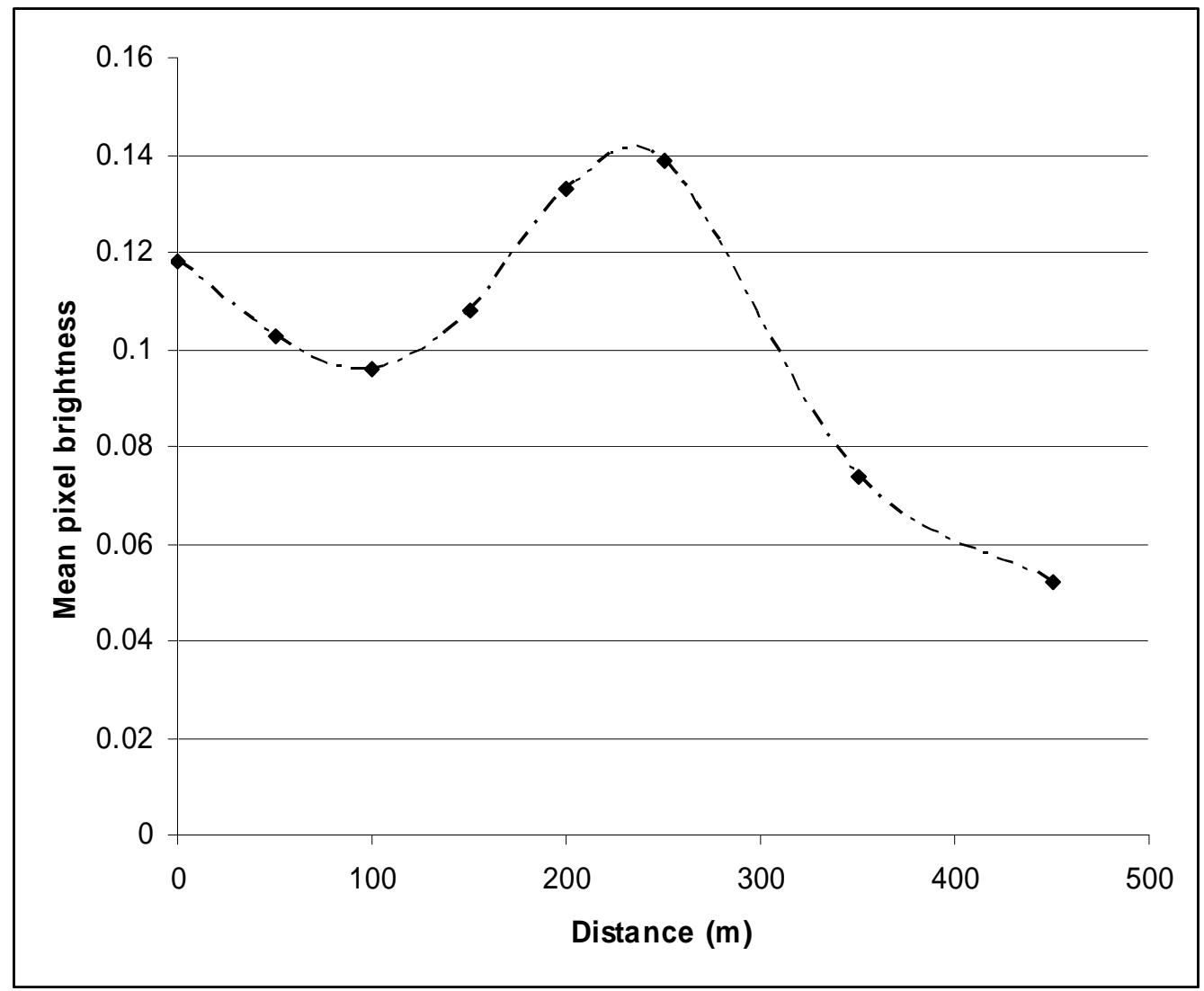

Figure 6 Graph to show the general trend in mean pixel brightness (proxy for amount of silt-sized sediment) on the soles of footwear over distance $(n=6)$ 


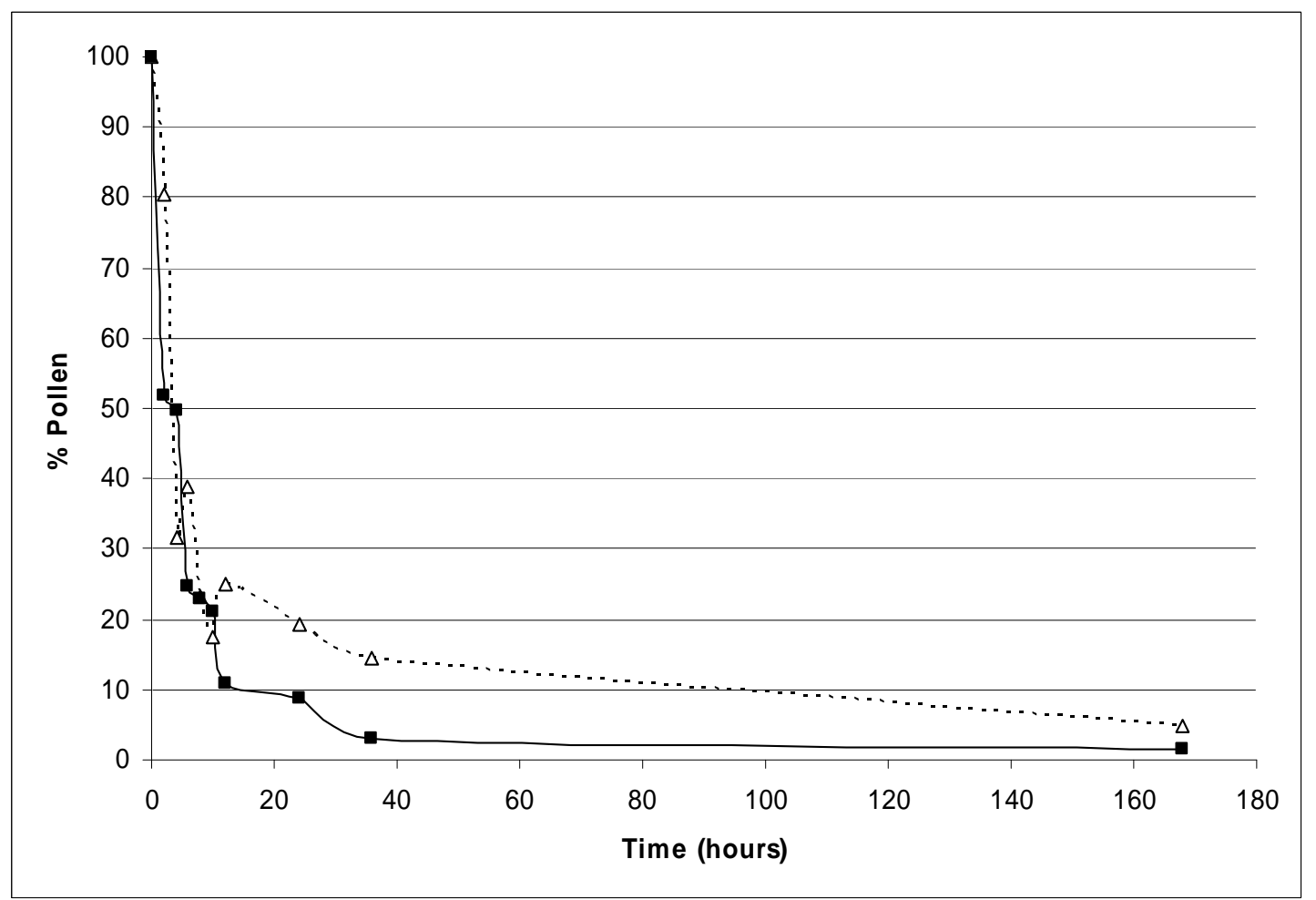

Figure 7a Graph to show the persistence of pollen particulates on the suede shoes over time.

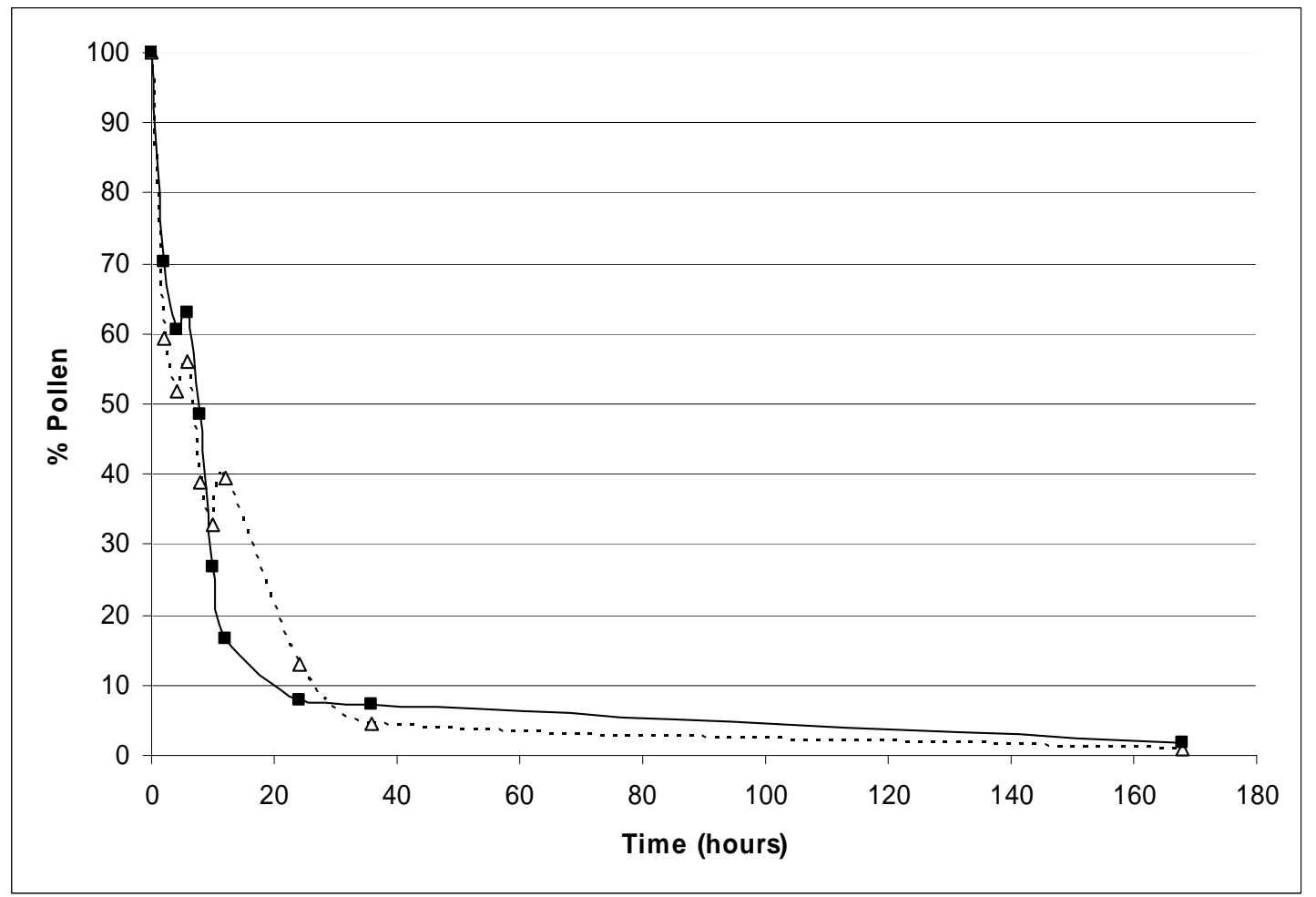

Figure $7 \mathrm{~b}$ Graph to show the persistence of pollen particulates on the cotton shoes over time. 